This item was submitted to Loughborough's Research Repository by the author.

Items in Figshare are protected by copyright, with all rights reserved, unless otherwise indicated.

\title{
The importance of dark adaptation for forensic examinations; an evaluation of the Crime-lite Eye ${ }^{\mathrm{TM}}$
}

PLEASE CITE THE PUBLISHED VERSION

https://doi.org/10.1016/j.scijus.2018.10.006

\section{PUBLISHER}

Elsevier () The Chartered Society of Forensic Science

VERSION

AM (Accepted Manuscript)

\section{PUBLISHER STATEMENT}

This paper was accepted for publication in the journal Science and Justice and the definitive published version is available at https://doi.org/10.1016/j.scijus.2018.10.006

\section{LICENCE}

CC BY-NC-ND 4.0

\section{REPOSITORY RECORD}

McMurchie, Beth, Roberto S.P. King, Paul Kelly, and George Torrens. 2019. "The Importance of Dark Adaptation for Forensic Examinations; an Evaluation of the Crime-lite Eye ${ }^{\text {TM". }}$ figshare.

https://hdl.handle.net/2134/35923. 


\title{
The importance of dark adaptation for forensic examinations; an evaluation of the Crime-lite Eye ${ }^{\mathrm{TM}}$
}

\begin{abstract}
Forensic practitioners are recommended to dark adapt their eyes prior to conducting evidential searches in the dark. The dark adaptation process remains poorly standardised across the discipline, with little quantified regarding the benefits of such preparative steps. Herein, we report the findings of a study that recruited 50 participants to assess the effectiveness of the Crime-lite Eye ${ }^{\mathrm{TM}}$, a darkness adaptation device developed to assist forensic practitioners both in the laboratory and in field. Participants were tasked with searching for the fluorescent signatures left by reaction of 1,8-diazafluoren-9-one (DFO) with amino acids, in a manner akin to the fluorogenic fingerprint treatment of porous evidence. Using an Epson Stylus Photo R265 inkjet printer, ink cartridges were filled with alanine solutions of various concentrations, allowing different motifs to be printed onto copy paper and subsequently developed using DFO. Participants searched for this 'evidence' both with and without dark adapted vision. On average, participants were able to locate and correctly recognise $16 \%$ more evidence once dark adapted using the Crime-lite Eye $^{\mathrm{TM}}$.
\end{abstract}

The increase in evidence located by participants once dark adapted suggests that crime scene officers should be dark adapting in order to visualise as much as possible. The time taken to dark adapt, 10 minutes on average during this study, is not excessively long, and should not significantly slow the investigation.

\section{Keywords}

Dark adaptation; crime scene; evidence; fingerprints; fluorescence; Crime-lite Eye.

\section{Introduction}

Forensic crime scene officers are often required to search for evidence in the dark, typically using a variety of light sources to locate fluorescent evidence that may be of interest. Laboratory fingerprint examiners are also required to work in the dark when searching for, and assessing the quality of, fingerprints that have been treated with a fluorogenic reagent (DFO or BY40, for example). For these investigations it is recommended that the practitioner should be fully dark adapted [1], to avoid the possibility of missing evidence. Full dark adaptation also allows investigators to more accurately judge fluorescent evidence, and determine whether it is worth investigating further [2,3]. 
Light and dark adaptation of the eyes is a well-known phenomenon, initially described in 1865 [4] and measured in 1903 [5]. Despite this, the dark adaptation process is still not fully understood [6]. Dark adaptation occurs in two parts: the first is rapid and due to cone function, the second is slower and due to rod function [7]. These two distinct functions form the classic dark adaptation curve, which remains similar between all individuals, with only slight differences [8]. The first part of dark adaptation is due to the cones and takes approximately three minutes to achieve. A large decrease in threshold luminescence is observed, which then slows and plateaus slightly before the rod function takes over, and this point of transition is known as the cone-rod break [9]. Once the rod function starts, there is a decrease in the curve again, before plateauing and reaching the absolute threshold. The threshold for dark adaptation is the point where the retina has reached absolute photochemical reactivity[8].

Whilst every person experiences the same biological process of dark adaptation, the time taken to reach the absolute threshold varies significantly between individuals [7]. Regardless of the difference in the time taken, each individual will go through the phase of fast cone adaptation followed by slower rod adaptation. Many factors will affect the time taken for the eyes to dark adapt, including eyesight and level of light adaptation prior to darkness exposure. Age has been shown to affect dark adaptation whereby older individuals require significantly more time to dark adapt than younger individuals [10]. An increase in the time taken to adjust to darkness has been shown to occur through both stages of cone and rod mediated dark adaptation in older individuals [10,11]. Gender, however, does not affect dark adaptation [12].

If the process of dark adaptation is critical to finding the maximum amount of evidence available, it is crucial that those looking for evidence are appropriately dark adapted. With so many variables affecting the time taken for an individual to achieve dark adaptation, it becomes difficult to establish a suitable time period in the dark to adapt, that doesn't waste valuable investigation time. Moreover, during investigations, it is common practice for investigators to leave the darkened area, or require the lights to be turned back on, thereby losing (or 'resetting') their current level of dark adaptation. A 2012 study by the Centre for Applied Science and Technology (CAST) showed that by wearing high wavelength longpass filtration goggles (red, $630 \mathrm{~nm}$ ) investigators were able to maintain some level of dark adaptation if leaving a darkened area. Additionally, if such goggles were worn in a lit environment prior to entering the dark, the dark adaptation time frame was reduced as individuals were effectively 'pre dark-adapting' [13].

A reliable method of monitoring an individual's level of dark adaptation would facilitate better control and allow a degree of standardisation during forensic investigations, thereby enabling more evidence to be visualised. The Crime-lite Eye $^{\mathrm{TM}}$, shown in Figure 1, is a commercially available darkness adaptation checker developed by Foster and Freeman, Evesham, UK, to give investigators a way of 
establishing/checking what level of dark adaptation they have reached. The Crimelite Eye offers a cheap and easy method of monitoring dark adaptation, costing approximately $£ 50$ to purchase. To date, however, the effectiveness of using a darkness adaptation device has not been evaluated within the scientific literature, nor has any quantitative data relating to the impact on finding evidence with and without dark adaptation been conducted.

The Crime-lite Eye ${ }^{\mathrm{TM}}$ uses a light emitting diode (LED) display that decreases in both numerical value as well as optical intensity from 20 to 1 . As illustrated in Figure 1 below, the number 20 appears relatively bright (when in a darkened environment with a degree of dark adaptation) with a gradual decrease in intensity to a very dim number 1 . As each person's eyes adapt to the dark at a slightly different rate and to a different endpoint, using such a device allows an adequate and repeatable level of dark adaptation to be achieved.
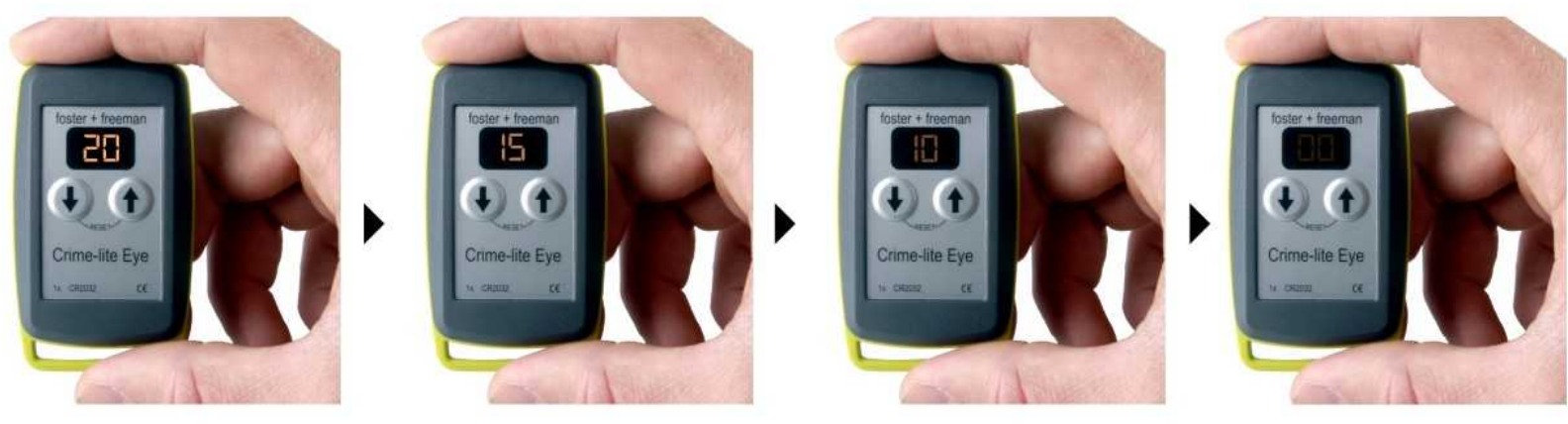

Figure 1 - The Crime-lite Eye ${ }^{\mathrm{TM}}$ showing a schematic representation of the dimming display as would be perceived during dark adaptation.

Many evidence types exist that possess inherent fluorescence (bodily fluids, fibres, explosives, etc.), with forensic light sources employed to help locate such traces. Fingermark examiners also use the power of fluorescence to their advantage when trying to improve contrast between the fingermark and the substrate on which it resides. There are a range of fluorogenic fingermark reagents recommended for use, many of which can be found in the Fingermark Visualisation Manual [1]. 1,8Diazafluoren-9-one (DFO) is a widely used Category A process that reacts with the amine group of amino acids, that are present within the fingerprint deposit itself, to yield a fluorescent product which fluoresces orange under green light excitation, with an excitation maxima at approximately $555 \mathrm{~nm}$ [14]. Other fluorescent processes work in a similar manner and require fluorescent screening, using appropriate light sources, in a darkened environment.

Obtaining consistent latent fingermarks, even from the same donor, proves to be effectively impossible due to the variance between both the components of a latent fingermark and the distribution of sweat that is deposited when the fingermark is left. 
Such inconsistencies between fingermark deposits can lead to variance in the emitted fluorescence of developed fingermarks when using enhancement reagents, such as DFO. In fingerprint research, the need to compare different fingerprint development techniques leads to many fingermarks being treated from numerous donors, this adds significant time to fingerprint research, alongside necessitating that many repeats are needed to ensure that the results accurate and reliable. To overcome this inconsistency in deposited fingermarks, a procedure has been described that employs an inkjet printer, filled with an artificial fingerprint sweat solution, to print artificial test fingermarks. The printing of artificial fingermarks, using an inkjet printer and a solution of amino acids and sodium chloride, was first reported by Schwarz [15]. Since this study, other research groups have attempted to optimise and tailor the printing method to more accurately resemble the components within natural fingerprint sweat $[16,17]$.

The study presented herein aims to highlight the effectiveness of the Crime-lite $\mathrm{Eye}^{\mathrm{TM}}$ in aiding the end user to achieve dark adaptation, with a specific reference to a quantifiable search for fluorescent fingermark evidence.

\section{Materials and Method}

To successfully determine the effectiveness of the Crime-lite Eye ${ }^{\mathrm{TM}}$, a standardised fluorescent test was established. The method of inkjet printing amino acids was utilised to create standardised fluorescent marks. Such a method not only allows 'fingerprints' to be printed and subsequently tested with enhancement reagents, but, as reported herein, other intricate and recognisable patterns/motifs may be produced that allow participants to conduct a fluorescent examination in a darkened environment, whilst allowing their observations to be duly recorded and scored. The number of different shapes, letters and patterns correctly visualised by each participant was recorded and the percentage of the total shapes, letters and patterns printed was calculated.

\subsection{Alanine Printing}

L-alanine was purchased from Sigma Aldrich and used to make a $0.4 \mathrm{mM}$ (A1) and $0.004 \mathrm{mM}$ (A2) solution of alanine in deionised water. Empty ink cartridges were purchased for an Epson Stylus Photo R265 inkjet printer and were washed with deionised water prior to use. The black and magenta ink cartridges were filled with $0.4 \mathrm{mM}$ (A1) or $0.004 \mathrm{mM}$ (A2) alanine solution, respectively. Various shapes, letters and patterns were subsequently printed onto two separate sheets of standard A4 white copier paper as shown in Figure 2. 
In addition to printing the patterns illustrated in Figure 2 in two different concentrations of alanine, the 'transparency' of some shapes was also altered on screen. The change in transparency did not affect the concentration of alanine that was printed (as the concentration remains stable and constant within each ink cartridge), it instead modified the amount of alanine deposited onto the paper by the printer. It was the intention to use the variable transparency printing method to mimic how fingermarks are deposited in 'real-world' scenarios, whereby some marks may be heavy and others quite light in both contact force and secretion level. The shapes printed at $75 \%$ transparency appear grey and pale pink on screen at $0.4 \mathrm{mM}$ and $0.004 \mathrm{mM}$ alanine concentrations respectively, compared to the 'standard' shapes ( $0 \%$ transparency), which appear black and magenta, as illustrated in Table 1 and Figure 2.

\begin{tabular}{|c|c|c|c|}
\hline $\begin{array}{c}\text { Alanine } \\
\text { Solution }\end{array}$ & Concentration & Transparency & $\begin{array}{c}\text { Colour seen } \\
\text { on screen }\end{array}$ \\
\hline A1 & $0.4 \mathrm{mM}$ & $0 \%$ & $\bullet$ \\
\hline $\mathrm{A} 1$ & $0.4 \mathrm{mM}$ & $75 \%$ & $\bullet$ \\
\hline $\mathrm{A} 2$ & $0.004 \mathrm{mM}$ & $0 \%$ & $\bullet$ \\
\hline $\mathrm{A} 2$ & $0.004 \mathrm{mM}$ & $75 \%$ & \\
\hline
\end{tabular}

Table 1 - The differences in concentration and transparency of the printed shapes, letters and patterns.
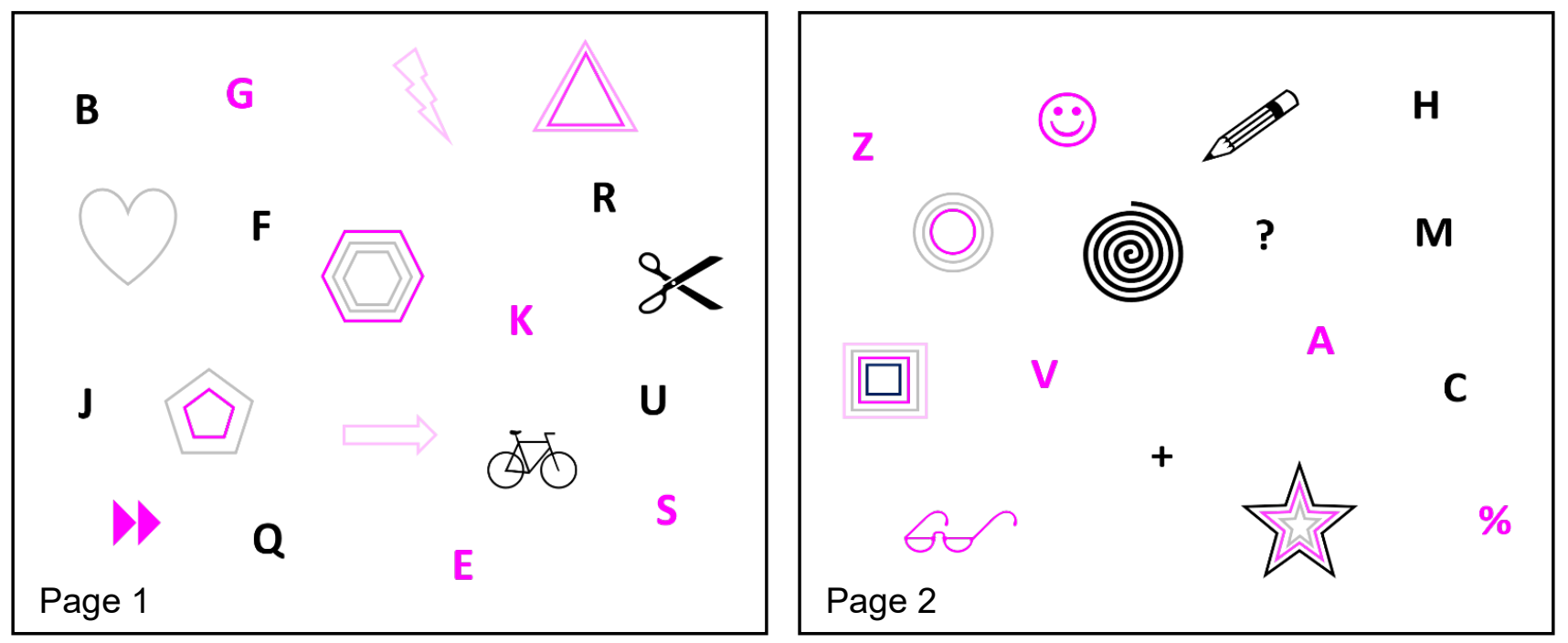

Figure 2 - The patterns as they appeared on screen prior to printing on two sheets of A4 copier paper, showing shapes, letters and patterns printed in A1 at $0 \%(\bullet)$, and $75 \%$ transparency $(\bullet)$ and $\mathrm{A} 2$ at $0 \%(\bullet)$ and $75 \%$ transparency $(\bullet)$.

1,8-Diazafluoren-9-one (DFO) (WA Products, $0.1 \mathrm{~g}$ ) was added to a solution of petroleum ether (Sigma Aldrich, $156 \mathrm{ml}$ ), acetic acid (VWR, $4 \mathrm{ml}$ ), ethyl acetate (Fisher Scientific, $20 \mathrm{ml}$ ) and methanol (Fisher Scientific, $20 \mathrm{ml}$ ) and stirred until all DFO had dissolved [18]. This solution was used to develop the printed alanine 
sheets. Each sheet was dipped into a tray containing the DFO working solution for approximately 5 seconds, left to air dry for approximately 2 minutes, then moved to an oven to dry/develop at $100{ }^{\circ} \mathrm{C}$ for 20 minutes. Whilst no visible pale pink colouring of the shapes and letters was visible to the naked eye, narrowband green (480-560 $\mathrm{nm}$ ) excitation using a Crime-lite 2 (foster+freeman) and viewing through an orange/red interference filter (OG590 longpass goggles) induced strong fluorescence. It was noted that visually, the brightness of the fluorescence varied between the two concentrations of alanine that were used and also between the $0 \%$ and $75 \%$ printing transparency values.

\subsection{Participant Dark Adaptation Study}

50 participants (31 males, 19 females) were recruited and informed of the study requirements before signing a written consent form. All participants were over 18 years of age. Participants were asked to sit in a darkroom, wearing orange/red filtered goggles (OG590) and using a green Crime-lite 2 (480-560 nm), both shown in Figure 3, they were asked to immediately speak out loud the letters, shapes and patterns that were visible to them on the two sheets of A4 paper (Figure 2). A Dictaphone was placed inside the darkroom to record the observations from each participant. Participants were instructed to look over the sheets fully once. Participants were asked to remain in their chair in the dark room, mainly to avoid the risk of injury in the dark, however they were free to lean as close to the two A4 sheets as required. The two A4 sheets were stuck to the wall one on top of the other, directly in front of the participants, a diagram showing the room set-up is shown in Figure 4. The room contained no windows, and the underside of the door was blocked to avoid excess light entering the room. However, the room was not completely blacked out, and very small amounts of light, for example from the gaps at the side of doors, was able to enter. This was intentional, as a dark room at a laboratory, or a room converted to a dark room at a crime scene is unlikely to be perfectly blacked out.

Once completed, participants were asked to leave the darkroom and let their eyes adapt to a fully lit environment for approximately 5 minutes, during which time an explanation into the use of the Crime-lite Eye ${ }^{\mathrm{TM}}$ was provided. It was ensured that all participants were out of the room for approximately the same length of time, however, as light adaptation is very quick compared to dark adaptation, participants eyes would be re-light adapted fully after 3 minutes.[8] 


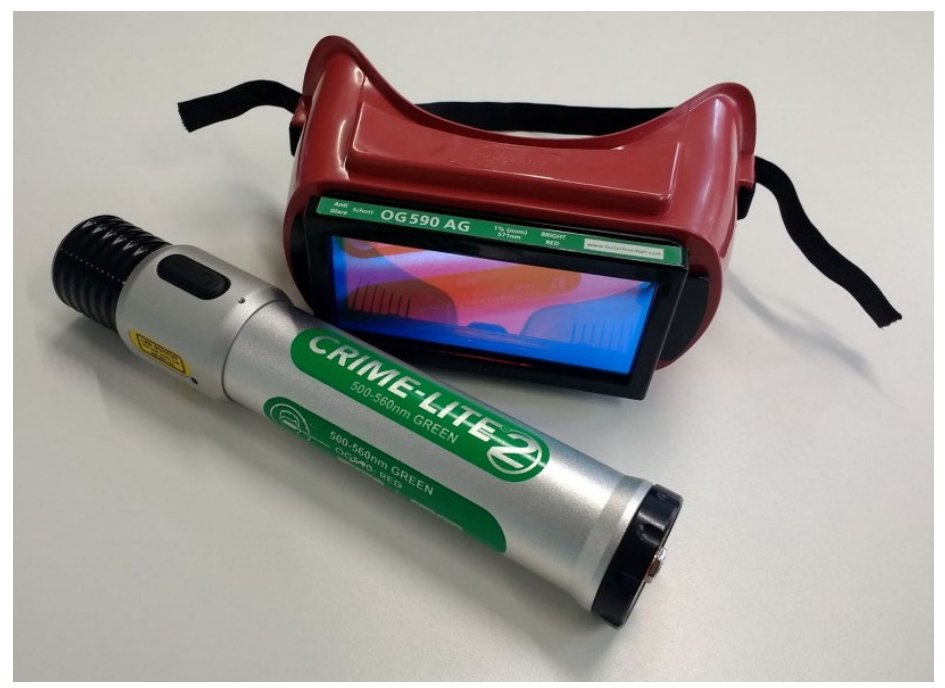

Figure 3 - The green Crime-lite 2 and orange/red filter goggles (OG590) used by participants throughout the study.

Participants were asked to re-enter the darkroom and use the Crime-lite Eye ${ }^{\mathrm{TM}}$ to establish adapted level of dark adaptation, which was duly recorded. Dark adaptation was reached with the Crime-lite 2 switched off. As explained in the introduction, each person's eyes are different and therefore able to reach a different number on the Crime-Lite $\mathrm{Eye}^{\mathrm{TM}}$; however participants were encouraged to reach the lowest number they thought possible, and say out loud which number they reached. Once the participant was dark adapted, the same light source and filtered goggles were used to examine the A4 samples again, with the Dictaphone recording the spoken observations as previously described.

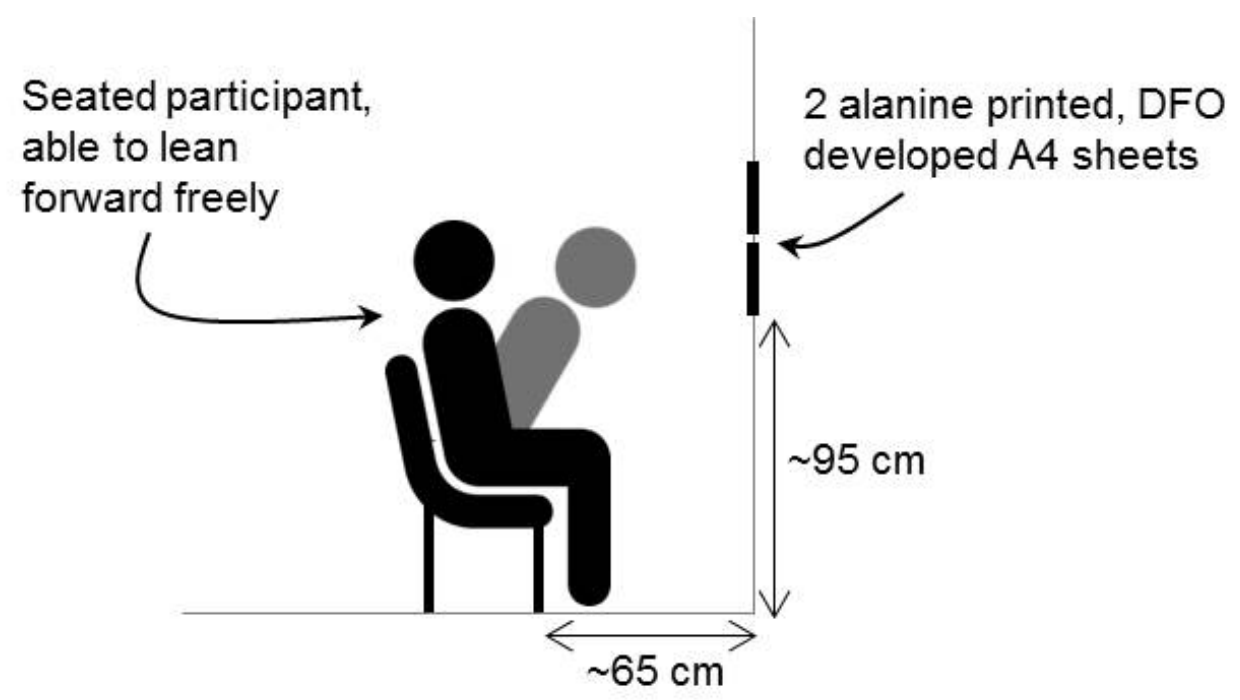

Figure 4 - The environmental set-up of the dark room, showing the position of the participant and the A4 sheets containing alanine printed, DFO developed shapes, letters and patterns. 


\section{Results}

The printed patterns that were developed with DFO provided good contrast against the substrate background. Several of the patterns printed and used within the study are shown below in Figure 5. The fluorescence of the patterns printed in A1 were more intense than the fluorescence of the patterns printed in A2, as shown below in Figure 5, comparing for example, the letters ' $S$ ' and ' $B$ '. A decrease in fluorescence intensity can also be seen between the shapes printed at $0 \%$ and $75 \%$ transparency.

(1)
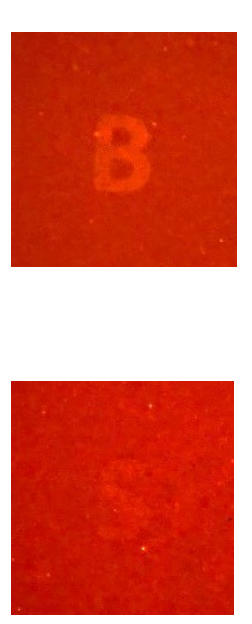

(2)

B
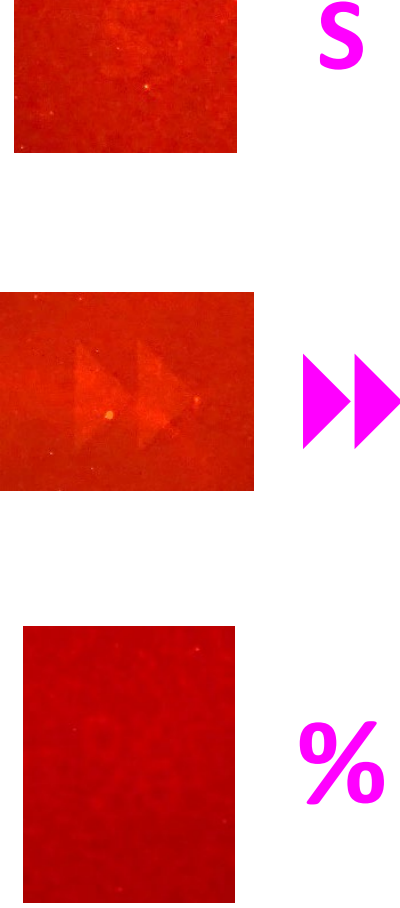

(1)
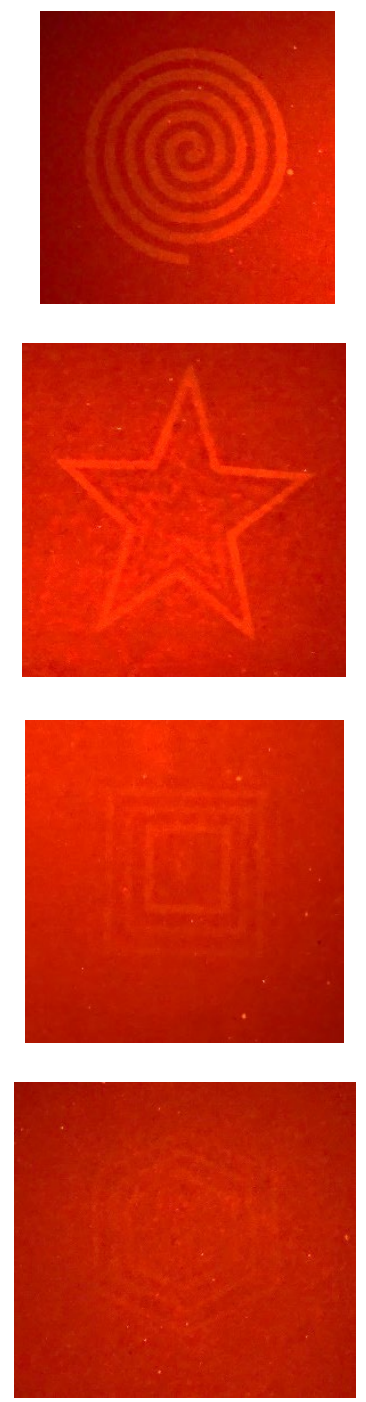
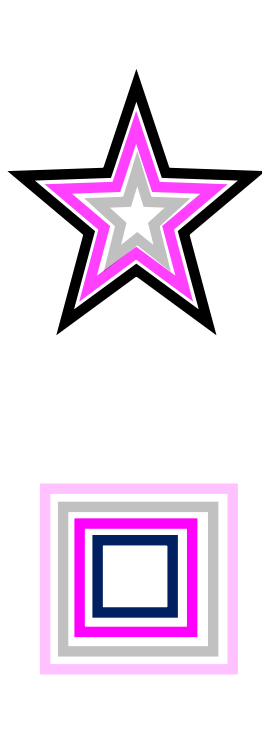

(2)
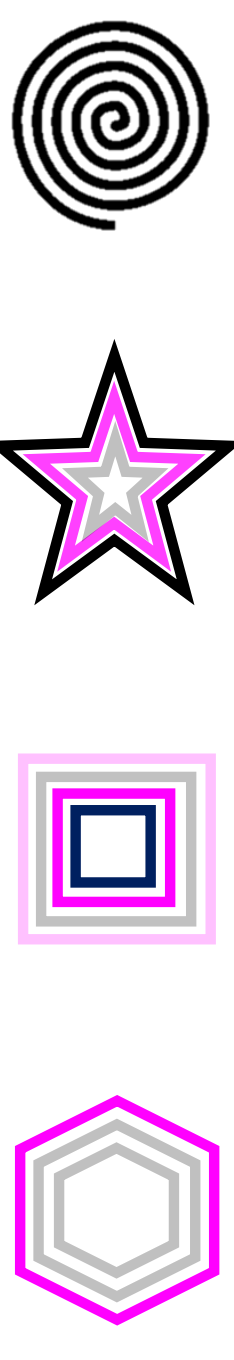

Figure 5 - Several of the patterns (1) printed using alanine, shown after development with DFO, photographed under green light excitation (490-560 nm) with an OG590 camera filter and (2) on screen pre-printing.

Participants waited an average of 10 minutes to dark adapt their eyes and most participants reached the number 5 on the Crime-lite Eye ${ }^{\mathrm{TM}}$. Of the 50 participants, 3 participants saw no change in the amount of shapes, letters and patterns visible pre- 
and post-dark adaptation, 1 participant saw a decrease of $2 \%$ in the amount of shapes, letters and patterns seen post-dark adaptation, however, the other 46 participants all saw an increase in the number and clarity of fluorescent features visible to them. Participants could see an average of $16 \%$ more evidence following dark adaptation, as illustrated in Figure 6.

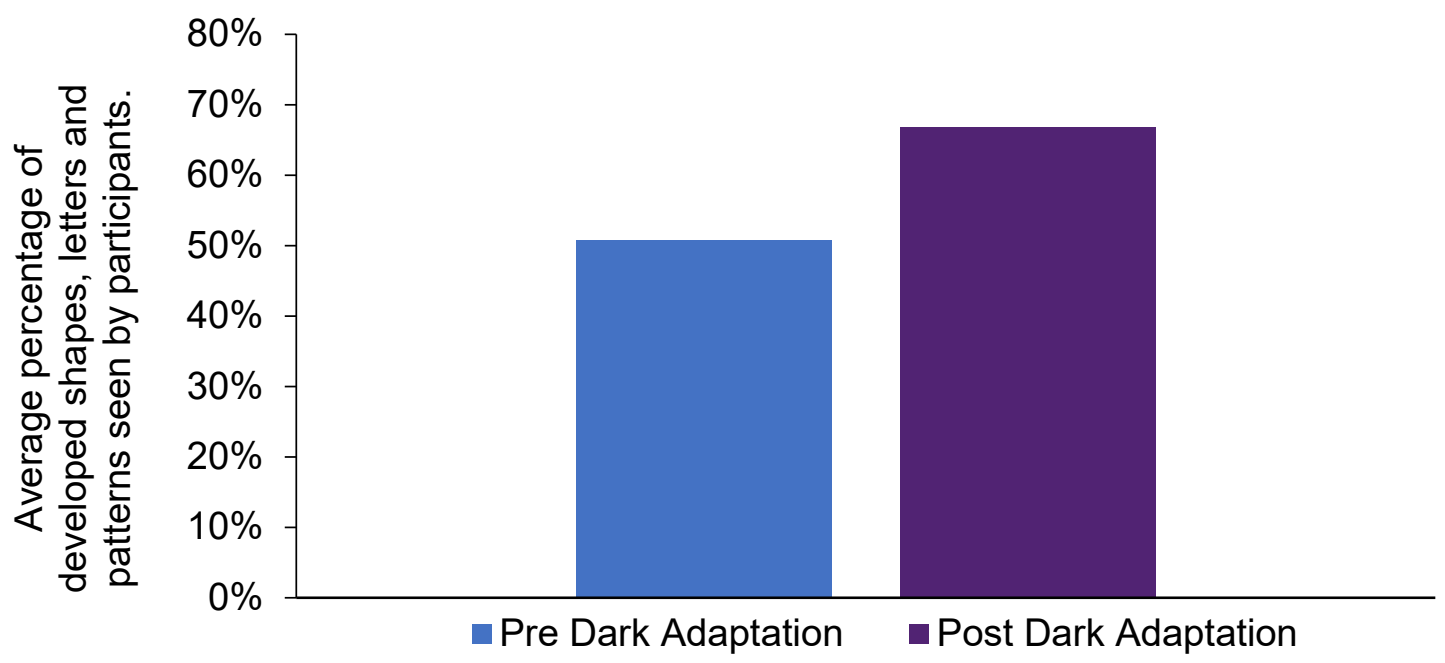

Figure 6 - The overall average percentage of shapes, patterns and letters seen by participants pre- and post-dark adaptation.

\section{Discussion}

The different marks visualised by the participants were analysed and it was noted that whilst each participant could see a different number of patterns, some shapes were easy to see compared to others which very few participants saw. For example, the spiral motif shown in Figure 2 and Figure 5 was seen by every participant, this was likely due to it being a large, basic and well-recognisable pattern that was positioned in a reasonably central location on the printed sheets. The spiral was also one of the patterns printed in A1 solution, so produced a higher fluorescent intensity compared to the $\mathrm{A} 2$ solution and the shapes printed at $75 \%$ transparency. There were two shapes that were not seen by any participant: the lightning bolt and the single right facing arrow. Both of these shapes were printed in A2 solution at $75 \%$ transparency, suggesting that this concentration and transparency combination was almost too faint to visualise using the light source in this study. However, some participants could see other shapes printed in A2 solution at $75 \%$ transparency, but only when they were part of a set of concentric shapes, such as the concentric squares shown in Figure 5. This suggests that participants could make out these faintest details when they were lead to them visually by brighter shapes in the same concentric pattern, however struggled to visualise these faint shapes when there was no way to know where they were. 
All letters were printed at $0 \%$ transparency, and most were visualised by the majority of participants. The average percentage of letters alone, seen following dark adaptation was $75 \%$, significantly higher than the $67 \%$ of all shapes, letters and patterns seen following dark adaptation. Whilst several different letters were printed in A2 solution, the letter ' $K$ ' was only seen by one participant. Participants generally saw less of the marks printed in A2 solution than the marks printed in A1 solution, however, the average percentage increase in marks seen following dark adaptation was $10 \%$ for both the marks printed in A1 solution and A2 solution. The average percentages of all marks seen by participants separated by solution concentration is shown below in Figure 7 .

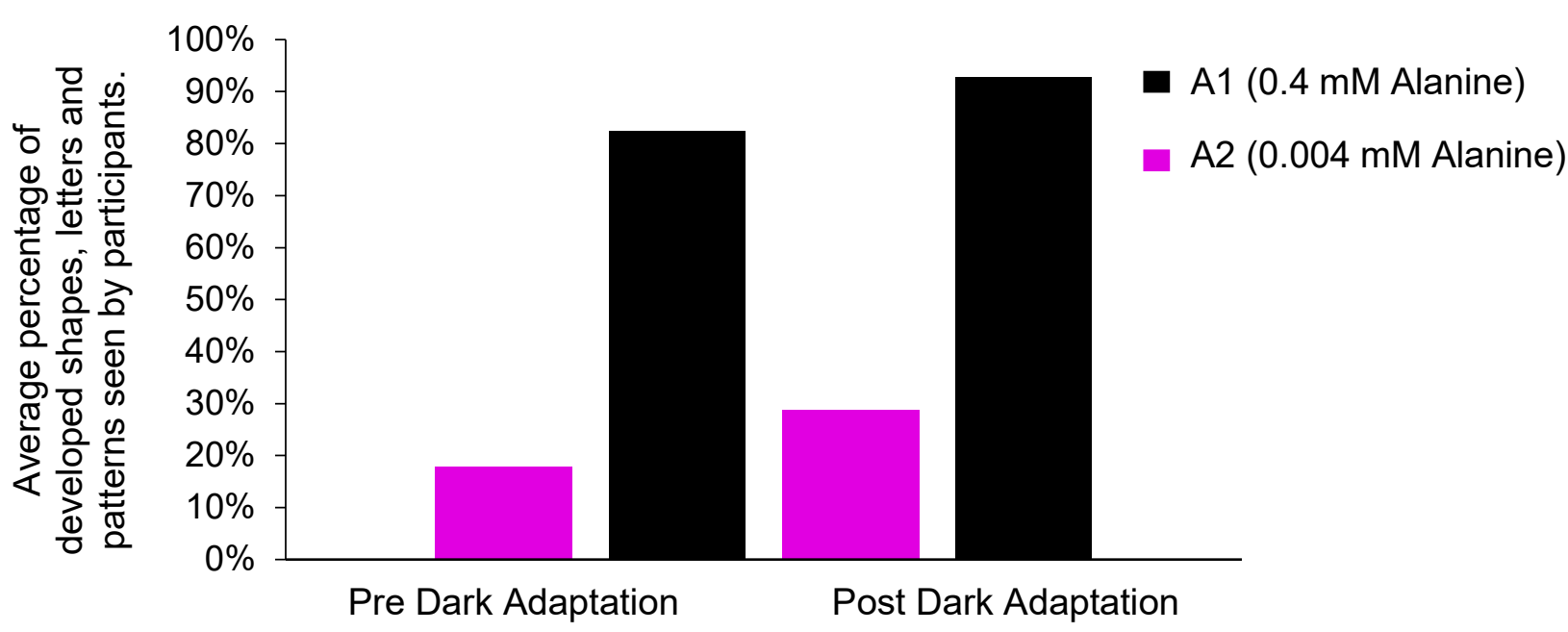

Figure 7 - The average percentage of shapes, patterns and letters seen by participants pre-and post-dark adaptation, separated by concentration of alanine.

Figure 7 illustrates that the increased number of fluorescent features observed by participants is consistent between those printed in A1 and A2 solutions. These observations suggest that the dark adaptation of participants helped increase their ability to find and accurately record more fluorescent evidence, independent of the fluorescent output intensity of the subject matter. This is echoed by the overall increase in fluorescent shapes, patterns and letters seen by participants, as shown in Figure 6 . This increase in ability to find not only more fluorescent marks, but also to find weaker marks, is of particular significance to forensic examiners, as it is often the weaker fluorescent evidence (fingermarks, body fluid stains, fibres, etc) that would be more likely missed.

Concentric shapes were used in the printed design as an attempt to mimic fine fingerprint ridge detail, as it is these ridges and minutiae that are required for identification. Participants could see $16 \%$ more shapes, letters and patterns after dark adaptation, but separating the results, participants could specifically see $21 \%$ more shapes after dark adaptation. This is due to an increase in not only amount of different shapes seen, but also in the number of concentric shapes counted. Looking specifically at an increase in concentric shapes seen by participants, participants on 
average saw $19 \%$ more shapes within each other following dark adaptation, the equivalent of finding 3 further concentric shapes of the 17 visible. This indicates that participants could more accurately count the 'ridges' once dark adapted.

Without dark adaptation, several participants mistook the letter ' $\mathrm{B}$ ' for the number ' 8 ', but every participant that mistook this letter initially, read it as the letter ' $B$ ' when viewing it following dark adaptation. One participant initially described this feature as looking "like an 8" when they were not dark adapted. Following dark adaptation, however, the same participant described the fluorescent feature as "clearly a B now, not an 8". Several other statements taken from the participant recordings are shown in Table 2, including when a correction was made, but also when participants were aware that more was visible to them compared to their initial viewing. This increase in accuracy, when dark adapted, is also vital to crime scene officers, as the ability to accurately identify evidence types is core to their role. The increase in accuracy also matches the conclusions already drawn suggesting that participants could see more concentric shapes once dark adapted, as they could distinctly visualise multiple shapes, rather than blurring them into only one legible shape. This again indicates that fine fingerprint details could be more accurately seen and counted after dark adaptation. Such comments by participants also helped confirm that participants were not simply remembering what they had seen on the sheet pre dark adaptation, as they had corrected themselves.

\begin{tabular}{|c|c|}
\hline $\begin{array}{c}\text { Participant } \\
\text { Number }\end{array}$ & Comments made \\
\hline 4 & "it's clearly a B now, it's not an 8" \\
\hline 9 & "missed lots the first time" \\
\hline 20 & "that's a H not an N" \\
"definite improvement"
\end{tabular}

Table 2 - Statements taken from participant post-dark adaptation recordings.

Only one participant said that they felt that the fluorescence was "less clear" when dark adapted. However, when reviewing which fluorescent features were visible to this participant before and after dark adaptation, they saw $2 \%$ more post-dark adaptation. This result, whilst lower than the average increase across all participants, was not a detrimental result as may have been implied from the participant's comments.

This study has only assessed the effect of dark adaptation when using green light $(490-560 \mathrm{~nm})$ viewed through a red/orange filter $(571 \mathrm{~nm})$, which is relevant to not only DFO examination, but also to marks developed using 1,2-indanedione. 
However, the fact that even at the red end of the spectrum, dark adaptation gives such an effect on the amount of marks visualised by participants, suggests that the benefit could be even greater when using reagents which require the blue end of the spectrum, such as luminol, as indicated during the study completed by CAST [13]. Further investigations into the effect of dark adaptation using different excitation and emission wavelengths would be of interest.

\section{Conclusions}

The results of this study show that participants can see more fluorescent DFO developed marks after using the Crime-lite Eye ${ }^{\mathrm{TM}}$ to establish a level of dark adaptation. Participants saw on average $16 \%$ more after becoming dark adapted. The results of this study also show that participants are not only able to see more on their second viewing, once dark adapted, but also that they are able to see things more accurately, identifying shapes and letters correctly that were misjudged on their initial viewing. This has good implications with fingerprint identification, as often fingerprint examiners are not only required to find fingerprints, but also to identify which fingerprints are useful for further examination. These results emphasise that fingerprint examiners should be dark adapted prior to investigation in the dark, and also shows that the Crime-lite Eye ${ }^{\mathrm{TM}}$ is a viable method for obtaining a known level of dark adaptation.

Further work investigating the effect of different wavelengths on the benefit of dark adaptation would improve the ability of forensic examination officers to be able to assess the appropriate level of dark adaptation necessary for their current examination. Additionally, repeating and expanding on the work done to assess the ability of red goggles to maintain dark adaptation between examinations would also greatly benefit forensic examiners.

\section{Declarations of interest}

None.

\section{Funding Source}

This research did not receive any specific grant from funding agencies in the public, commercial, or not-for-profit sectors.

\section{References}

[1] H.L. Bandey, S.M. Bleay, V.J. Bowman, R.P. Downham, S. V G, Fingermark Visualisation Manual, 2014. 
http://www.officialpublicationsonline.co.uk.libproxy.abertay.ac.uk/publications/d ownload/9781782462347.

[2] K. Knight, Fingerprints Don't Increase Friction, J. Exp. Biol. 212 (2009) i-i. doi:10.1242/jeb.033977.

[3] O. Jay, G. Havenith, Finger skin cooling on contact with cold materials: An investigation of male and female responses during short-term exposures with a view on hand and finger size, Eur. J. Appl. Physiol. 93 (2004) 1-8. doi:10.1007/s00421-004-1146-x.

[4] H. Aubert, Physiologie der Netzhaut, (1865) 394.

[5] H. Piper, Über Dunkeladaptation, Zeitschrift Für Psychol. Und Physiol. Der Sinnesorgane. 31 (1903) 161. http://echo.mpiwgberlin.mpg.de/ECHOdocuView?url=/permanent/vlp/lit32940/index.meta.

[6] T.D. Lamb, E.N. Pugh Jr, Dark adaptation and the retinoid cycle of vision, Prog. Retin. Eye Res. 23 (2004) 307-380. doi:10.1016/j.preteyeres.2004.03.001.

[7] S. Hecht, C. Haig, A.M. Chase, The Influence of Light Adaptation on Subsequent Dark Adaptation of the Eye., J. Gen. Physiol. 20 (1937) 831-50. doi:10.1085/jgp.20.6.831.

[8] M. Pirenne, Dark-Adaptation and Night Vision, in: H. Davson (Ed.), Eye. Vol. 2 Vis. Process, Academic Press, London, 1962: pp. 93-122.

[9] V. Lakshminarayanan, Light Detection and Sensitivity, in: J. Chen, W. Cranton, M. Fihn (Eds.), Handb. Vis. Disp. Technol., Springer-Verlag, Berlin, 2011: pp. 86-90. doi:10.1007/978-3-540-79567-4.

[10] A.J. Gaffney, A.M. Binns, T.H.T.H. Margrain, Aging and cone dark adaptation, Optom. Vis. Sci. 89 (2012) 1219-1224. doi:10.1097/OPX.0b013e318263c6b1.

[11] G.R. Jackson, C. Owsley, G. Mcgwin, Aging and dark adaptation, Vision Res. 39 (1999) 3975-3982. doi:10.1016/S0042-6989(99)00092-9.

[12] M. Rowe, Differences between the sexes in sensory sensitivity and performance in the visual and auditory modalities, 1983.

[13] H. Bandey, Adapting for Forensic Examinations in the Dark, in: Fingerpr. Soc. Conf., 2016.

[14] S.M. Bleay, R.S. Croxton, M. De Puit, Fingerprint Development Techniques, John Wiley \& Sons Ltd, Singapore, 2018.

[15] L. Schwarz, An amino acid model for latent fingerprints on porous surfaces, J. Forensic Sci. 54 (2009) 1323-1326. doi:10.1111/j.1556-4029.2009.01168.x.

[16] M. Hildebrandt, J. Sturm, J. Dittmann, Printing artificial sweat using ink jet printers for the test set generation in forensics: an image quality assessment of the reproducibility of the printing results, Image Qual. Syst. Perform. X. 8653 (2013) 1-10. doi:Unsp 86530olr10.1117/12.2004526.

[17] S. Hong, I. Hong, A. Han, J.Y. Seo, J. Namgung, A new method of artificial latent fingerprint creation using artificial sweat and inkjet printer, Forensic Sci. Int. 257 (2015) 403-408. doi:10.1016/j.forsciint.2015.10.005.

[18] Centre of Applied Science and Technology, Fingerprint Source Book, United Kingdom Home Off. (2013) Chapter 10-11. https://www.gov.uk/government/uploads/system/uploads/attachment_data/file/ 143865/fsb-chap3-sec10and11-development.pdf. 